\title{
Neural responses to perturbations in visual and auditory metronomes during sensorimotor synchronization
}

\author{
Daniel C Comstock $^{1}$, Ramesh Balasubramaniam ${ }^{1}$
}

${ }^{1}$ Cognitive and Information Sciences, University of California, Merced, CA, 95343, USA

Address for Correspondence:

Ramesh Balasubramaniam, $\mathrm{PhD}$

Sensorimotor neuroscience laboratory, Cognitive \& Information Sciences University of California, Merced 5200 N Lake Road Merced CA 95343

$\mathrm{Ph}: 209.228 .2314$

ramesh@ucmerced.edu 


\begin{abstract}
Tapping in synchrony to an isochronous rhythm involves several key functions of the sensorimotor system including timing, prediction and error correction. While auditory sensorimotor synchronization (SMS) has been well studied, much less is known about mechanisms involved in visual SMS. By comparing error correction in auditory and visual SMS, it can be determined if the neural mechanisms for detection and correction of synchronization errors are generalized or domain specific. To study this problem, we measured EEG while subjects tapped in synchrony to separate visual and auditory metronomes that both contained small temporal perturbations to induce errors. The metronomes had inter-onset intervals of 600 milliseconds and the perturbations where of 4 kinds: $+/-66$ milliseconds to induce period corrections, and +/- 16 milliseconds to induce phase corrections. We hypothesize that given the less precise nature of visual SMS, error correction to perturbed visual flashing rhythms will be more gradual than with the equivalent auditory perturbations. Additionally, we expect this more gradual error correction will be reflected in the visual evoked potentials. Our findings indicate that the visual system is only capable of more gradual phase corrections to even the larger induced errors. This is opposed to the swifter period correction of the auditory system to large induced errors. EEG data found the peak N1 auditory evoked potential is modulated by the size and direction of an induced error in line with previous research, while the P1 visual evoked potential was only effected by the large late-coming perturbations resulting in reduced peak latency. Looking at the error response EEG data, an Error Related Negativity (ERN) and related Error Positivity $(\mathrm{pE})$ was found only in the auditory +66 condition, while no ERN or $\mathrm{pE}$ were found in any of the visual perturbation conditions. In addition to the ERPs, we performed a dipole source localization and clustering analysis indicating that the anterior cingulate was active in the error detection of the perturbed stimulus for both auditory and visual conditions in addition to being involved in producing the ERN and $\mathrm{pE}$ induced by the auditory +66 perturbation. Taken together, these results confirm that the visual system is less developed for synchronizing and error correction with flashing rhythms by its more gradual error correction. The reduced latency of the P1 to the visual +66 suggests that the visual system can detect these errors, but that detection does not translate into any meaningful improvement in error correction. This indicates that the visual system is not as tightly coupled to the motor system as the auditory system is for SMS, suggesting the mechanisms of SMS are not completely domain general.
\end{abstract}




\section{Introduction}

Tapping in synchrony to a rhythmic stimulus like a metronome involves the use of several key components of the sensorimotor system including time, prediction, and error correction. Finger tapping has been widely used to study sensorimotor functions and abilities, especially with auditory sensorimotor synchronization (Repp, 2005). Behavioral studies of finger tapping have contributed to our understanding of how movement trajectories contribute to error correction in motor timing (Balasubramaniam et al, 2004; Hove et al., 2014). Recently, neuroimaging techniques have also been used to build understanding of the neural basis of error correction in sensorimotor synchronization (SMS) using EEG (Praamstra et al., 2003; Jang et al., 2016) By studying the neural processes involved in visual and auditory SMS, the two modalities can be compared, and thus tested to see to what extent the neural mechanisms of SMS are modality specific or generalized.

To understand the differences between auditory and visual SMS we must first understand the differing capabilities between the two. One of the largest differences is the greater variability of the timing of taps with visual SMS (Repp, 2005). In addition to the differences in tapping variability, there are different limits to the tempo at which a stimulus can be entrained to; an auditory metronome can be synchronized to an interonset interval (IOI) as low as 100ms, while the lower IOI limit for accurate visual synchronization to a flashing stimulus is around 500ms (Repp, 2005). Even though there are clear differences in synchronization ability between the visual and auditory domains, it remains to be seen exactly why those differences exist.

Another important aspect of synchronizing movements to rhythmic stimulus is error detection and correction. Monitoring of the timing of each stimulus and of the synchronized movements is necessary to ensure continued synchronization. Since any movement action takes 
time from initiation to completion, the timing of each stimulus must be predicted in advance (Chen et al, 1998). The prediction of the onset of each oncoming event then allows for a comparison of the predicted timing with the actual timing for error detection in the stimulus. Errors of synchronization must be monitored for in addition to errors in the stimulus before error correction can occur. To study the nature of error correction in SMS, occasional temporal perturbations in an otherwise isochronous stimulus have been used to induce errors (Thaut et al., 1998; Repp, 2000, 2001), and a two-level system of error correction has been put forward (Vorberg \& Wing, 1996). The models posit that error correction falls into two types: Period correction and Phase correction. A period correction occurs in response to a large, noticeable error in timing, and involves updating a central time keeper. A phase correction takes place in response to a small error in timing that is below the conscious threshold and is thought to involve a more peripheral adaptive process (Repp, 2001; Repp \& Keller, 2004).

To understand the neural mechanisms involved in error correction in SMS, previous work on auditory error correction has shown a modulation of the auditory-evoked potentials believed to modulate attention in response to errors in the timing of an otherwise isochronous auditory rhythm (Tecchio et al., 2000; Praamstra et al., 2003). The auditory evoked potentials, in this case the auditory P1 and N1, have shown that both the direction of the induced error, and the magnitude of the error modulate the components (Praamstra et al., 2003). In addition to the sensory evoked potentials, error induced potentials have been found in response to synchronization errors caused by perturbing the timing of a metronome (Praamstra et al, 2003). The error related components, the Error Related Negativity (ERN) and associated Error Related Positivity $(\mathrm{Pe})$ have been shown to be indicative of detection of response errors, allowing for another measure of the error response (Yeung et al, 2004). 
This study explores the differences in auditory and visual SMS error correction, as well as the correlating neural substrates. By measuring EEG while synchronizing finger taps with separate auditory and visual flashing metronomes, both with occasional timing errors, we can measure behavioral and neural differences between the two sense modalities. We hypothesize that since the visual system does not facilitate the same temporal precision in synchronizing to a visual flashing metronomes as the auditory system facilitates with an auditory metronome that error correction in the visual system will be a more gradual phase correction, even for larger perturbations. We further expect this reduced error correction ability to be reflected in a diminished modulation of the visual evoked components compared to the auditory evoked components, as well as reduced error response components.

\section{Materials and methods}

\subsection{Participants}

Ten subjects participated in the experiment (6 females; ages 18 to 34). All participants were right handed. Data from 4 additional subjects were collected but not included in analysis because they were unable to synchronize with the visual stimulus. All participants had normal hearing and normal or corrected vision. Participants gave informed consent after the experimental procedures where explained. This study was approved by the Institutional review board (IRB) for research ethics and human subjects.

\subsection{Task}

Participants were asked to tap in synchrony to separate auditory and visual metronomes with an inter-onset interval (IOI) of $600 \mathrm{~ms}$. The $600 \mathrm{~ms}$ interval (standard IOI) was chosen because a faster visual metronome is difficult for most people to synchronize to (Repp \& $\mathrm{Su}$, 2013). In both sequences, there were occasional perturbations of the duration of the IOI. There 
were four types of perturbations; increasing the standard IOI by $16 \mathrm{~ms}$, or by $66 \mathrm{~ms}$; and decreasing the standard IOI by $16 \mathrm{~ms}$, or by $66 \mathrm{~ms}$. The intervals were chosen based on the Praamstra et al. (2003) protocol and increased to scale with the larger IOI (600 ms compared to $500 \mathrm{~ms}$ ), and due to the limitations of the $60 \mathrm{~Hz}$ monitor used in the study.

The experiment was split into the auditory condition and the visual condition, with a counterbalanced design so half of the subjects did the auditory condition first, and half did the visual condition first, but never on the same day. Each half of the experiment consisted of 120 blocks, with each block consisting of sequences of 50 stimuli with a minimum of 3 seconds between each block. Each sequence contained 4 perturbations, with the perturbation in a given sequence always of one type. The temporal location of the perturbations was varied to avoid being predictable, with a minimum of 9 non-perturbed stimuli between perturbations. Subjects were given a 10-minute break at the halfway point of each condition. The experiment began with applying the EEG cap after written consent was obtained. Subjects were then given written instructions for the experiment, and performed one practice block that contained shifts of each type before starting.

The auditory stimuli consisted of $50 \mathrm{~ms} 1000-\mathrm{Hz}$ pure tones with a $10 \mathrm{~ms}$ rise time and $30 \mathrm{~ms}$ fall time presented through headphones at a comfortable volume. The visual stimuli consisted of a $50 \mathrm{~ms}$ gray flash on a black screen. For both conditions, subjects faced a monitor that had a black screen and a gray fixation point that remained constant. Tapping was performed with the index finger of the right hand on a metal plate attached to a Makey Makey input device that records tapping by sending a small electrical signal to an output lead that the subject holds on their left hand. An input lead for the Makey Makey was then attached to a metal plate that the subject tapped. When the subject touched the metal plate, it completed a circuit in the Makey 
Makey which sends the signal to the computer to indicate a tap (Collective \& Shaw, 2012).

Subjects performed the task while seated in a comfortable chair.

\subsection{EEG data acquisition and processing}

EEG was continuously recorded with an ANT-Neuro 32 electrode cap with electrodes placed according to the 10-20 International electrode system and recorded at $1024 \mathrm{~Hz}$. The EEG data were uploaded and processed with EEGLAB (Delorme \& Makeig, 2004), and the ERP data processed using ERPLAB (Lopez-Calderon \& Luck, 2014). ERP data was preprocessed by first down sampling to $256 \mathrm{~Hz}$, then applying a high pass filter with $6 \mathrm{db}$ cutoff at $.1 \mathrm{~Hz}$, followed by a low-pass filter with a $6 \mathrm{db}$ cutoff at $56.25 \mathrm{~Hz}$ to eliminate $60 \mathrm{~Hz}$ line noise. Data were then examined and any bad sections removed by hand. Any bad channels were detected and removed using the probability measure within the ASR plugin for EEGLAB that compares channels with their surrounding channels (Mullen et al., 2015). The data were then re-referenced to the linked mastoids, and then ICA was performed using the infomax algorithm within EEGLAB (Bell \& Senjnowski, 1995). Following ICA, the component data were examined and eye blink and eye movement components were rejected to clean the data of further artifacts. Data were epoched using ERPLAB centered around the onset of the perturbed stimulus and centered on the participants taps that corresponded to the perturbed stimuli. Each epoch was from -.5 seconds of the event onset to 1 second past the onset. In addition to removing blink and eye movement components, any epoch that had an eye blink during the stimulus onset was removed.

For source localization, the EEG data down sampled to $256 \mathrm{~Hz}$ before being filtered with a high pass filter with a $6 \mathrm{db}$ cutoff at $.5 \mathrm{~Hz}$ and a low pass filter with a $6 \mathrm{db}$ cutoff at $56.25 \mathrm{~Hz}$. As specified in the earlier section, data were then examined again and bad sections removed by 
hand. Any bad channels were detected and removed using the probability measure within the ASR plugin for EEGLAB that compares channels with their surrounding channels. The data were then re-referenced to the linked mastoids. Then ICA was performed using the infomax algorithm within EEGLAB. Dipole source localization was performed using the Dipfit2 plugin that performs source localization by fitting an equivalent current dipole model using a non-linear optimization technique using a 4-shell spherical model (Kavanagk et al., 1978; Scherg, 1990). Data were epoched the same as with the ERP data. All components that had dipoles located outside of the brain model were rejected, as were all components with a dipole residual variance of greater than $15 \%$. The epoched data were then clustered using a PCA method in EEGLAB with the K-means algorithm with the clustering based solely on the location of the equivalent dipoles for each component. The data were clustered into 12 clusters for both the stimuluslocked and response-locked data as that number fits closest to achieving 1 independent component per subject per cluster.

\section{Results}

\subsection{Behavioral data}

To analyze the average tap time asynchrony induced by the perturbations, we ran the taptime asynchronies from $\mathrm{T}-3$ to $\mathrm{T}+6$ for each perturbation condition and for both auditory and visual modalities in a within-subjects, repeated measures ANOVA. To get the tap time asynchrony values we first normalized the baseline by taking the average tap time asynchrony from T-4 to T-1 and subtracting it from the T-3 to T+6. This procedure was used by Repp (2001) and was done to reduce inter-subject variability due to differing negative mean asynchronies since we are not interested in those inter-subject differences. The Auditory and Visual conditions were first analyzed separately with 3 factors: Direction (positive vs negative), Magnitude (66 ms 
vs $16 \mathrm{~ms}$ ), and Position (the 10 levels of position: $\mathrm{T}-3$ to $\mathrm{T}+6$ ). These data are displayed in figure 1, where a large change in the amount of asynchrony can be seen after the perturbation at point $\mathrm{T}$ for each condition.
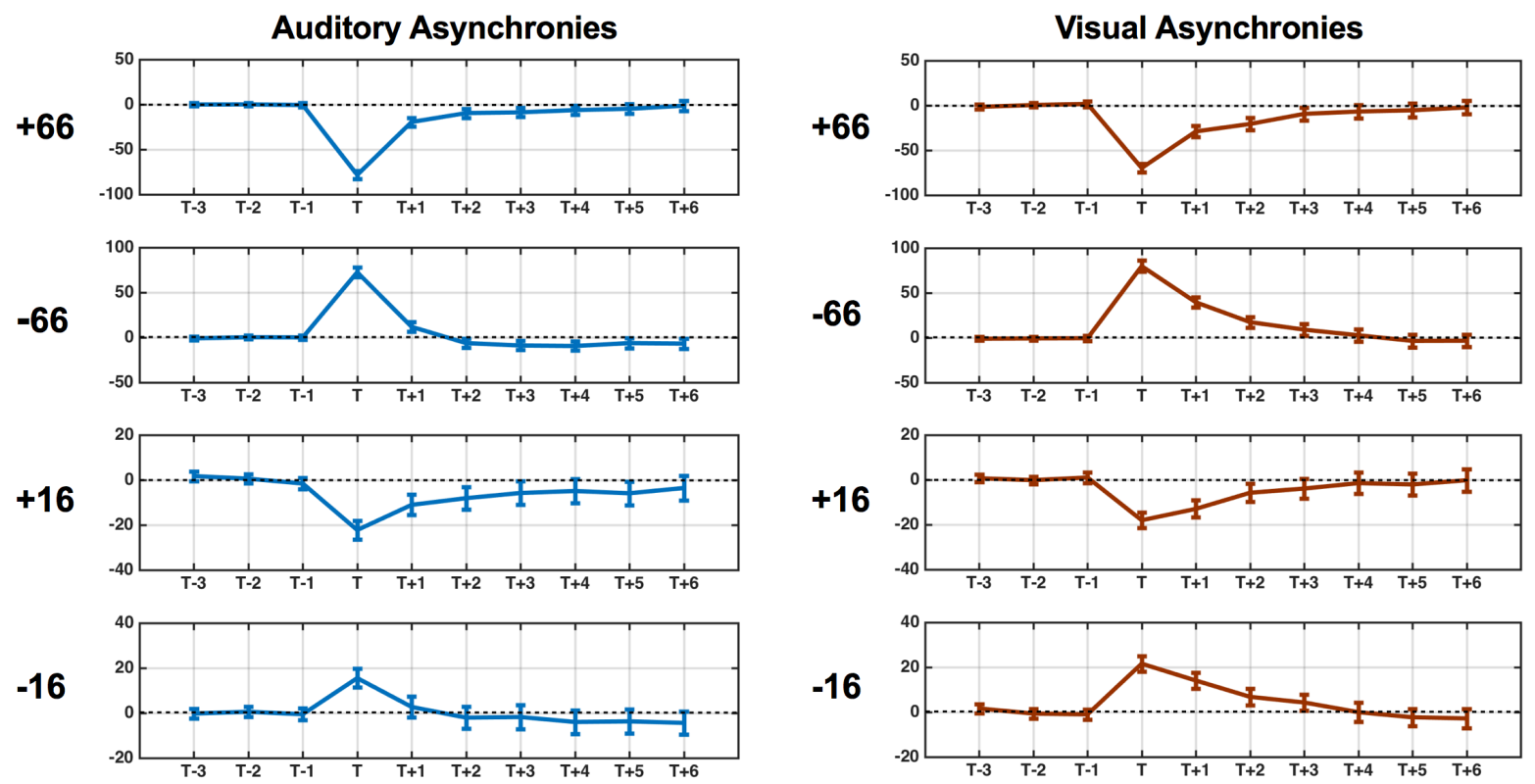

Figure 1. Tap asynchronies at each stimulus position. The baseline asynchrony was normalized to 0 for all subjects and indicated by the dashed lines. Error bars show standard error. Note that the scale of the y axes are different between the 16 and $66 \mathrm{~ms}$ perturbations

Similar to the results of Praamstra et al. (2003), we do not see a significant main effect of Magnitude or of Position in the Auditory condition. We do see an effect in Direction $F(1,9)=$ $51.11, \mathrm{P}<0.001$, since the direction of the asynchrony changes with the direction of the perturbation. Additionally, there is an interaction between Direction and Position $\mathrm{F}(9,81)=$ $62.62, \mathrm{P}<.001$ which is due to the large change in asynchrony at the position of the perturbation, $\mathrm{T}$, in a direction determined by the direction of the perturbation. We also find an interaction between Direction and Magnitude $F(9,81)=7.43, \mathrm{P}<.05$. In the visual condition we find no significant main effect of either Magnitude or Direction, but we do see a main effect for 
Direction $\mathrm{F}(9,81)=62.62, \mathrm{P}<.001$ as well as interactions for Direction and Position $\mathrm{F}(9,81)=$ 78.94, $\mathrm{P}<.001$, and for Direction and Magnitude $\mathrm{F}(9,81)=33.89, \mathrm{P}<.001$.

In order to compare the results between the auditory and visual conditions we ran a within-subjects repeated measures ANOVA on the combined auditory and visual tap-tone asynchrony data using 4 factors: Direction, Magnitude, Position, and Modality (auditory and visual). In this analysis, we found no significant main effects on Magnitude or Position. We found an effect of Direction F $(1,9)=204.36, \mathrm{P}<.001$, interactions between Direction and Position $\mathrm{F}(9,81)=425.71, \mathrm{P}<.001$, and between Direction and Magnitude $\mathrm{F}(9,81)=55.01, \mathrm{P}<$ .001 . This analysis also found a main effect of Modality $\mathrm{F}(1,9)=11.48, \mathrm{P}<.01$, which is a result of the more gradual corrections of the visual perturbations (interaction of Modality X Position $\mathrm{F}(9,81)=2.03, \mathrm{P}<.05)$, and of the greater tendency for overcorrection in the Auditory negative perturbations and under-correction in the Auditory positive perturbations (interaction of Modality by Direction $\mathrm{F}(1,9)=5.46, \mathrm{P}<.05)$.

In order to check if the corrections were happening more quickly for the larger perturbations, and to check if the direction effected the speed of the correction, the tap-time asynchrony data were normalized from time points $\mathrm{T}$ to $\mathrm{T}+6$ for both Auditory and Visual conditions (figure 2). These data were analyzed using the same procedure and factors as the taptime asynchrony data. The Auditory normalized asynchronies were spread out due to the amount of overcorrection in the negative perturbations and under-correction in the positive overcorrections yielding a main effect of Direction $\mathrm{F}(1,9)=23.98, \mathrm{P}<.001$. This under and over correction subsequently washed out any significant effects on Magnitude or Position. There was an interaction between Direction and Magnitude F $(1,9)=12.42, \mathrm{P}<.01$, likely driven by the effect of Direction. The Visual normalized asynchronies show only a main effect of Position 
$\mathrm{F}(5,45)=26.76, \mathrm{P}<.001$ as the correction was uniformly changing and only effected by

Position. The clear difference between the Auditory and Visual normalized asynchronies is shown by the main effect of Modality F $(1,9)=13.65, \mathrm{P}<.001$ when an analysis of the two conditions combined were run. Additionally, the combined analysis shows a main effect of Direction $\mathrm{F}(1,9)=24.23, \mathrm{P}<.001$, and interactions between Direction and Magnitude $\mathrm{F}(1,9)=$ 11.08, $\mathrm{P}<.01$ and Modality and Direction $\mathrm{F}(1,9)=16.15, \mathrm{P}<.001$.
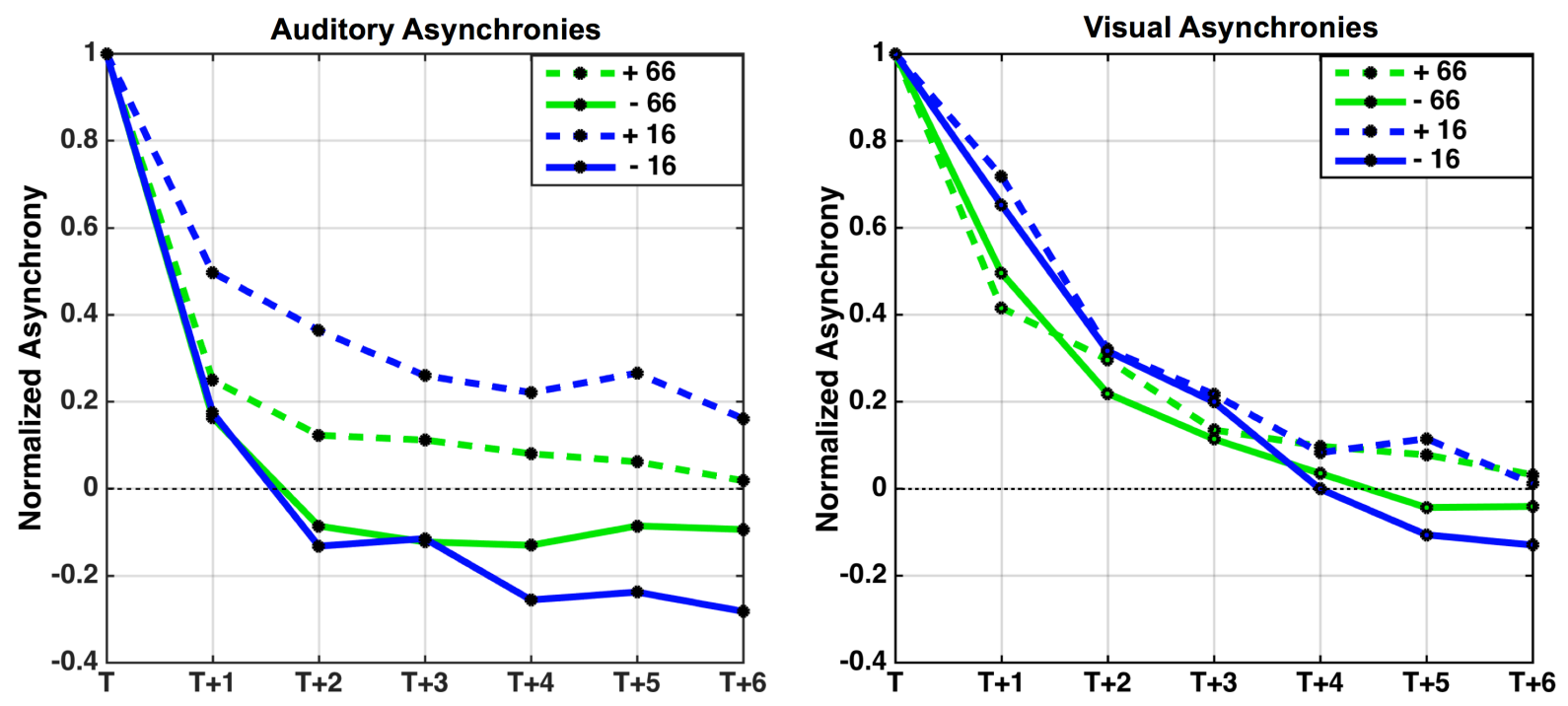

Figure 2. Normalized asynchronies for both auditory and visual conditions. The visual condition induced a nearly uniform relative correction while the auditory condition shows a separation between conditions primarily due to under and overcorrections.

\subsection{ERP waveforms}

We focused on several different waveforms in studying the neural underpinnings of error correction, starting with the auditory evoked potentials shown in averaged waveforms from Fz in figure 3 . The stimulus locked waveform shows a P1 component peaking around $100 \mathrm{~ms}$ post stimulus followed by a negative going waveform (N1) around $130 \mathrm{~ms}$, and then the positive P2 component peaking near $200 \mathrm{~ms}$ post stimulus. Additionally, in the -66 condition there was a second negative peak following the $\mathrm{N} 1$ around $170 \mathrm{~ms}$ which may be a mismatch negativity 
response (MMN). For the purposes of the current study, we focused on the auditory N1 component, which showed the strongest deviation in the +66 condition.
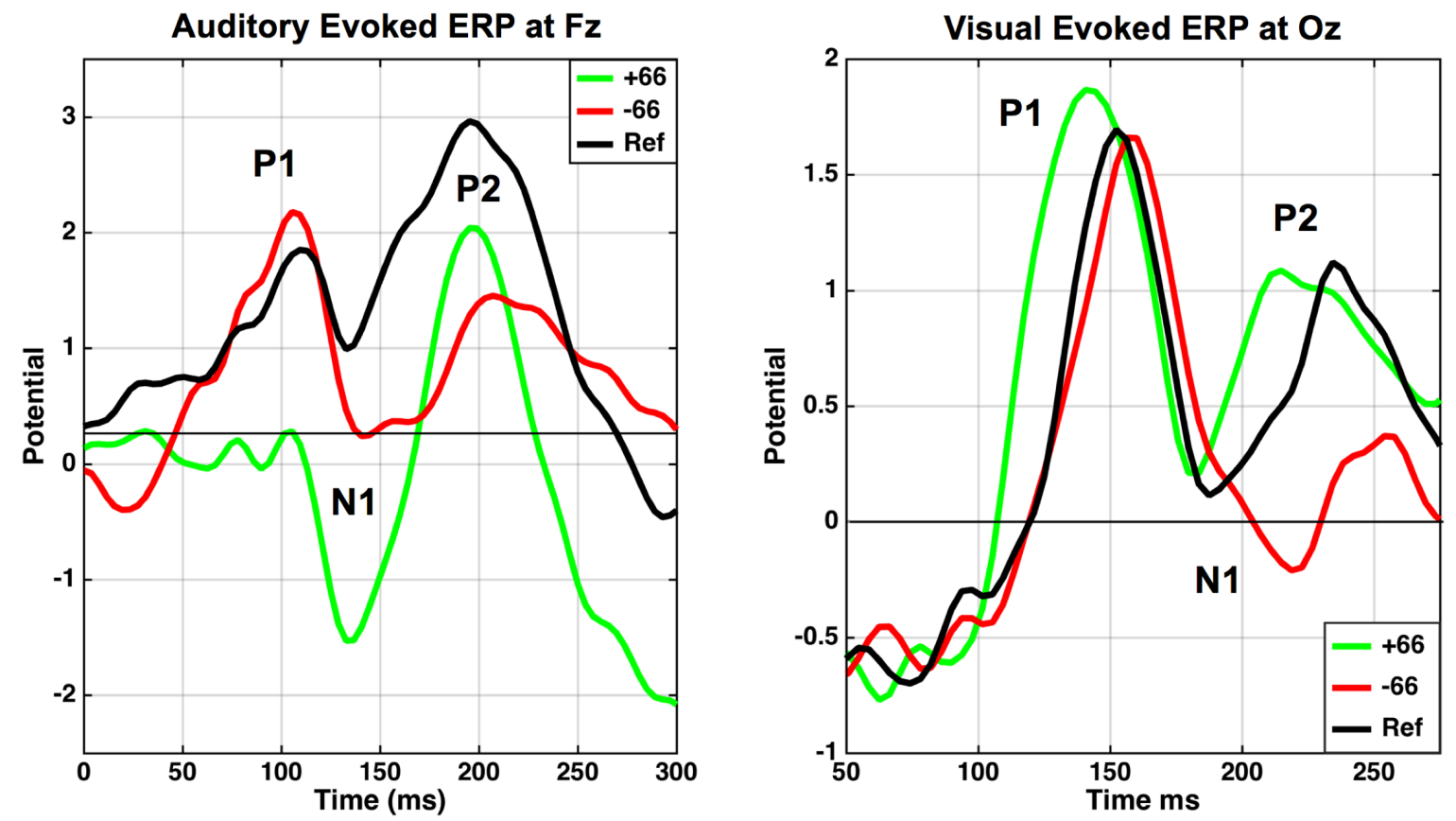

Figure 3. Grand average Auditory and Visual evoked ERPs at Fz and Oz, respectively. The Auditory evoked ERPs are stimulus locked to the onset of a tone, while the Visual evoked ERPs are stimulus locked to a flash. Both plots show wave forms evoked by +66 perturbations, the -66 perturbations, and a non-perturbed stimulus.

To look at the visual evoked potentials seen in figure 3, we used the averaged waveform at $\mathrm{Oz}$ instead of at $\mathrm{Fz}$, as this is the point where the primary visual attentional components can be best measured from. The wave forms consist of a large positive peak near $150 \mathrm{~ms}$ post stimulus (P1) followed by the negative N1 at around $180 \mathrm{~ms}$. There is then a second positive waveform peaking between 200 and $250 \mathrm{~ms}$ labeled the P2. This analysis focuses on the visual P1 wave, which like the auditory N1 was effected the most in the +66 condition.

The response evoked potentials, shown in figure 4, are measured at Fz. They consist of a large negative premovement wave followed by a large positive postmovement wave near 100 ms. Following Praamstra et al. (2003), we focus on the ERN, which would occur around $200 \mathrm{ms,}$ 
and the associated Error Positivity (Pe) near $300 \mathrm{~ms}$. Since the tap time asynchrony was in the range of $-50 \mathrm{~ms}$, there is some overlap with the frontal stimulus evoked components. This is especially pronounced in the visual response locked waveform as there is a large P3 component that overlaps with the expected onset of the ERN and Pe (Figure 8). Since the P3 component is stimulus locked, but the response locked waves are locked to the tap time, the effect is a large positive wave in the visual response locked ERP not seen in the auditory response locked ERP. Since the expected stimulus time onset differs with the actual stimulus onset time in the perturbation conditions, the effect of the stimulus evoked waveforms on the response evoked waveforms is temporally shifted based on the size and direction of the perturbation.

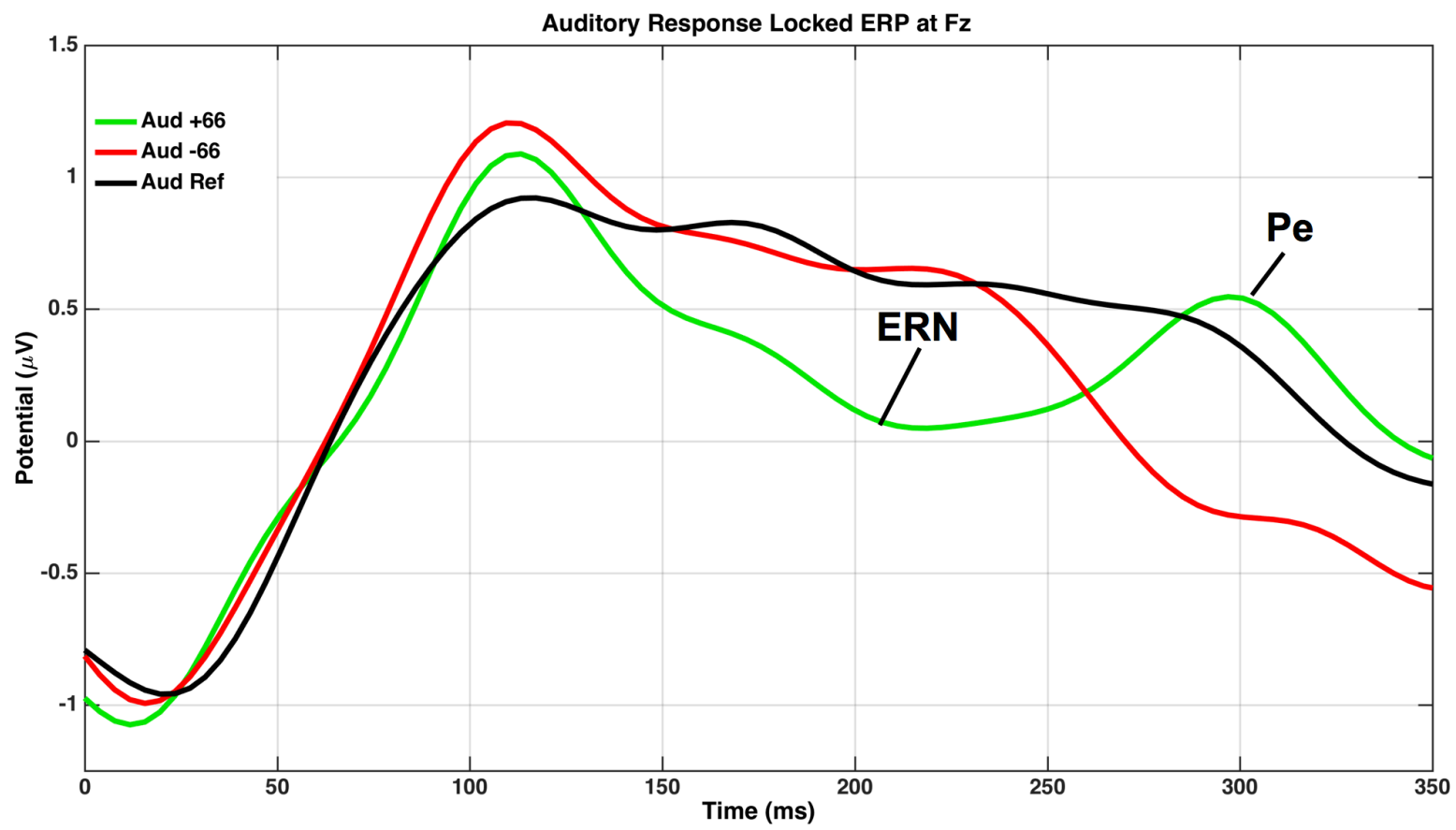

Figure 4. Auditory ERP at Fz time-locked to the tap onset for $+/-66 \mathrm{~ms}$ Perturbations and a non-perturbed reference. 


\subsection{Auditory-evoked potentials}

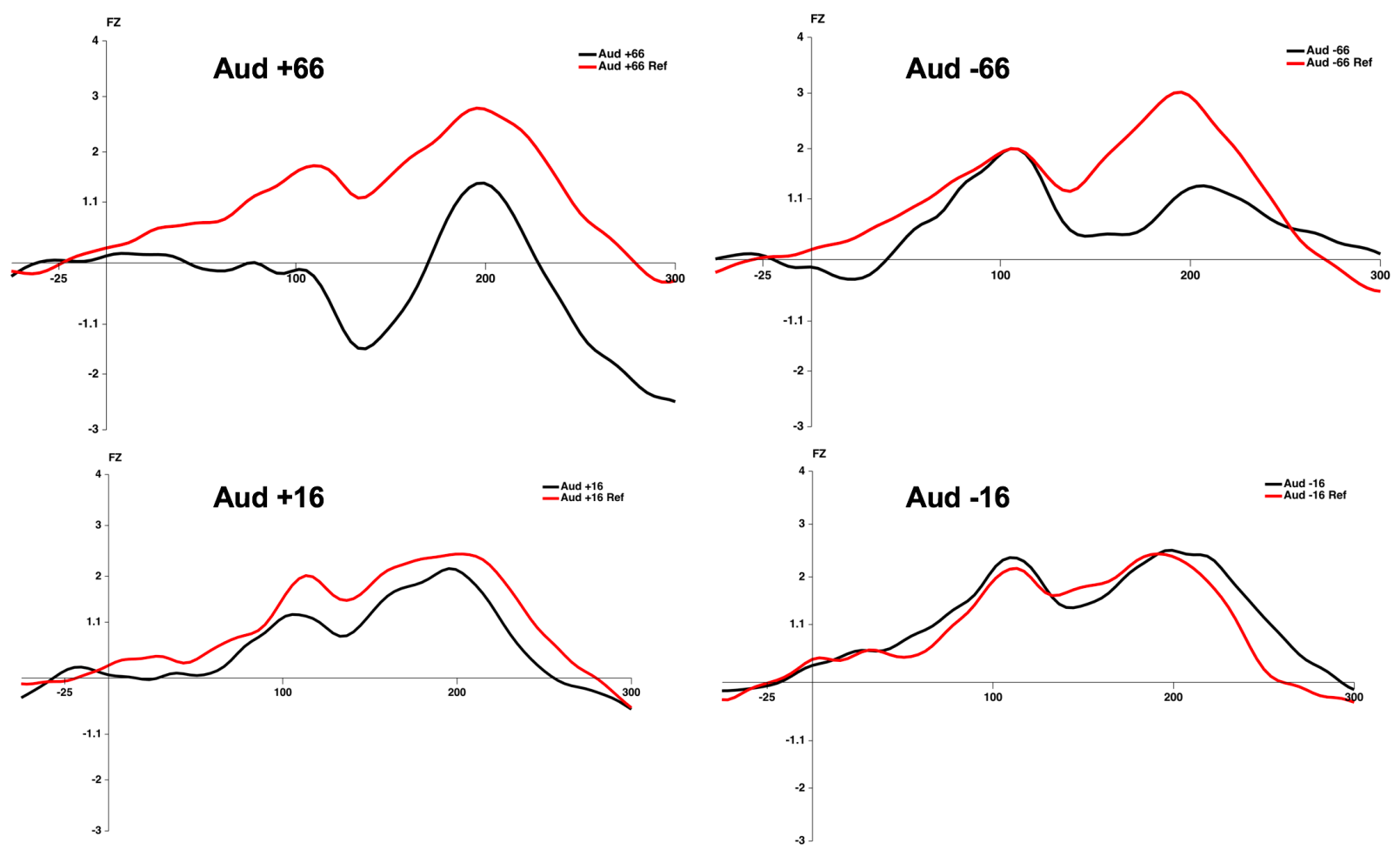

Figure 5. Auditory evoked potentials at Fz for both the perturbed conditions at time $\mathrm{T}$, and corresponding reference (non-perturbed) condition at T-2 for each of the 4 perturbation types

The AEPs were analyzed based on the difference in peak amplitude of the N1 wave between the perturbation conditions and the corresponding non-perturbation reference condition seen in figure 5 . The perturbation condition was at $\mathrm{T}$, while the reference condition was always at T-2, and taken from the same blocks as its corresponding perturbation. The peak amplitude was measured using a local peak finding procedure within ERPLAB between 110 and 160 ms post stimulus onset using a jackknife procedure. The jackknife procedure takes as many grand averages as there are subjects, with each grand average subtracting one subjects' waveform. Applying this method has the advantage with non-linear measures, such as peak amplitude and peak latency, of reducing error variance, effectively reducing the probability of a Type II error while not increasing the likely-hood of a Type I error (Luck, 2014). These difference scores were 
then entered into a 2 factor within-subjects, repeated-measures ANOVA with the factors of Direction (+/- perturbations) and Magnitude (66 and 16). This yielded main effects of Magnitude $(1,9)=325.58, \mathrm{P}<.001$, and Direction $(1,9)=566.89, \mathrm{P}<.001$, indicating that the N1 wave was modulated by both the direction and magnitude of the perturbations, and was concurrent with Praamstra et al (2003). Additionally, there was an interaction between Magnitude and Direction $(1,9)=297.37, \mathrm{P}<.001$, due to the $66 \mathrm{~ms}$ perturbations having much larger effects than the $16 \mathrm{~ms}$ perturbations. These results are largely driven by the $66 \mathrm{~ms}$ perturbations and especially the +66 perturbation which produced the most deviant N1.

\subsection{Visual-evoked potentials}

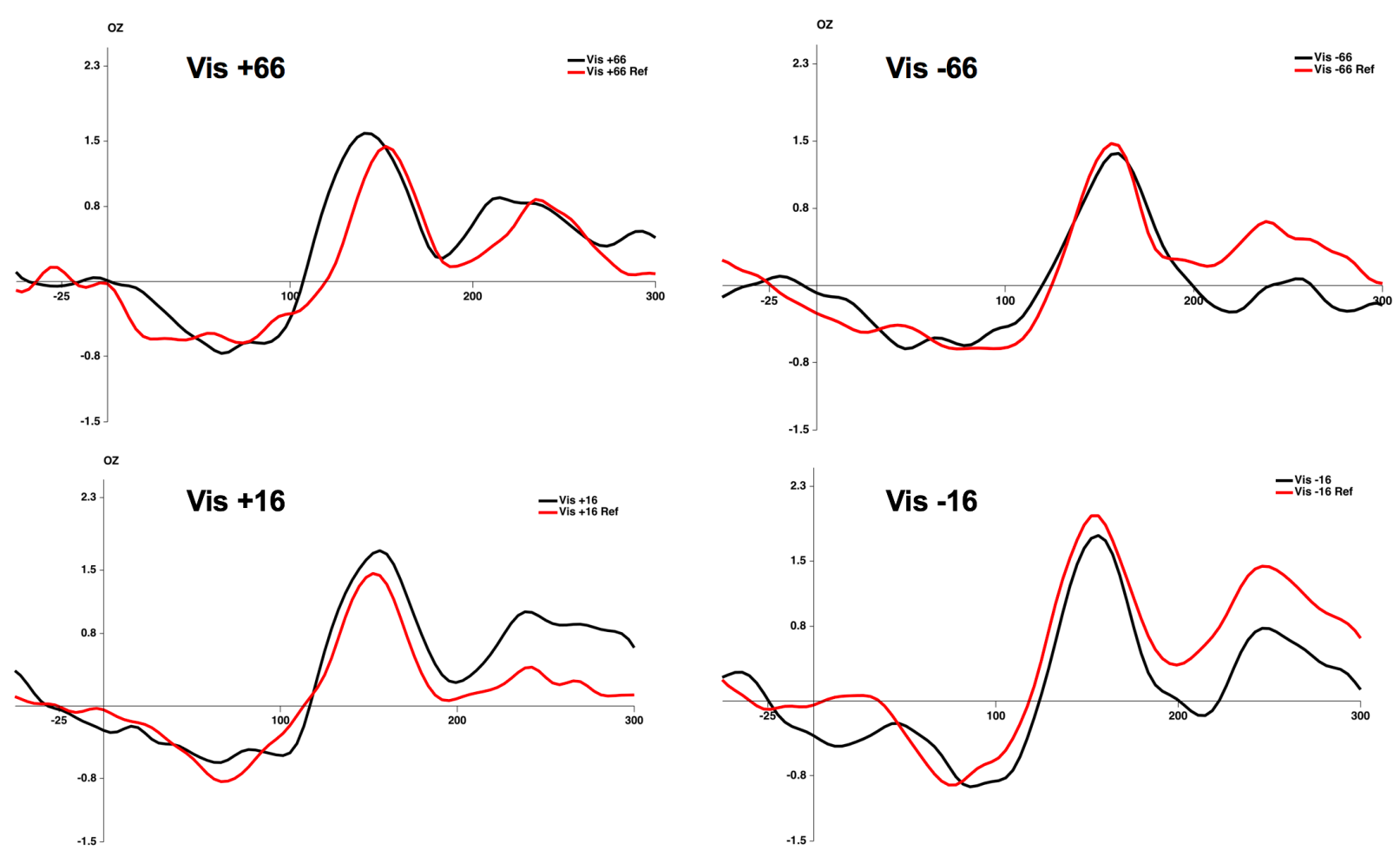

Figure 6. Visual evoked potentials at $\mathrm{Oz}$ for both the perturbed conditions at time $\mathrm{T}$, and corresponding reference (non-perturbed) condition at $\mathrm{T}-2$ for each of the 4 perturbation types 
The VEPs were analyzed based on the difference in peak amplitude of the P1 wave between perturbation conditions and their corresponding references in the same fashion as the auditory N1 was analyzed. The peak was measured using the local peak function in ERPLAB between 100 and 190ms post stimulus onset as seen in figure 6. The 2-way ANOVA showed a main effect of Direction $(1,9)=107.67, \mathrm{P}<.001$, and an interaction effect between Direction and Magnitude $(1,9)=114.49, \mathrm{P}<.01$, but no significant main effect of Magnitude. These results are likely driven by the +66 perturbation which resulted in a P1 wave of reduced latency. To test the differences in latency, additional analyses were performed using the differences between the local peak latency of the P1 scores of the perturbed and corresponding reference P1 waves. This analysis was done using a jackknife procedure and the 2-way ANOVA, showing significant main effects in both Magnitude $(1,9)=48.96, \mathrm{P}<.001$, and Direction $(1,9)=36.42, \mathrm{P}<.001$, as well as in the interaction between Magnitude and Direction $(1,9)=50.8, \mathrm{P}<.001$. These results confirm that the P1 wave had a reduced latency in the +66 condition.

\subsection{Response-locked potentials}

The response-locked potentials from the auditory condition in figure 7 were measured at Fz. An inspection of Figure 7 reveals a waveform similar to that as reported in Praamstra et al (2003). To analyze the ERN, we measured the mean-amplitude between 180 and $220 \mathrm{~ms}$ of the waveforms and then took the difference of the mean-amplitude between the perturbation condition and the corresponding reference from a non-perturbed tap at $\mathrm{T}-2$. Those difference scores were entered into a within-subjects, repeated measures 2-way ANOVA with the factors of Direction and Magnitude. We found no significant effects which is in contrast to the results reported by Praamstra et al (2003). The Pe that usually corresponds to the ERN, and appears in 
the +66 auditory condition was analyzed in the same way as the ERN, except using a meanamplitude window of 300 to $400 \mathrm{~ms}$. Here we find a main effect of Direction $(1,9)=10.66, \mathrm{P}<$ .05 and a significant interaction between Direction and Magnitude $(1,9)=8.44, \mathrm{P}<.05$, but no main effect of Magnitude. Since the Pe almost always corresponds to an ERN, these results suggest that the ERN was lost due to the confound of the auditory-evoked N1 and P2 waves, while the Pe was not effected due to its later onset.

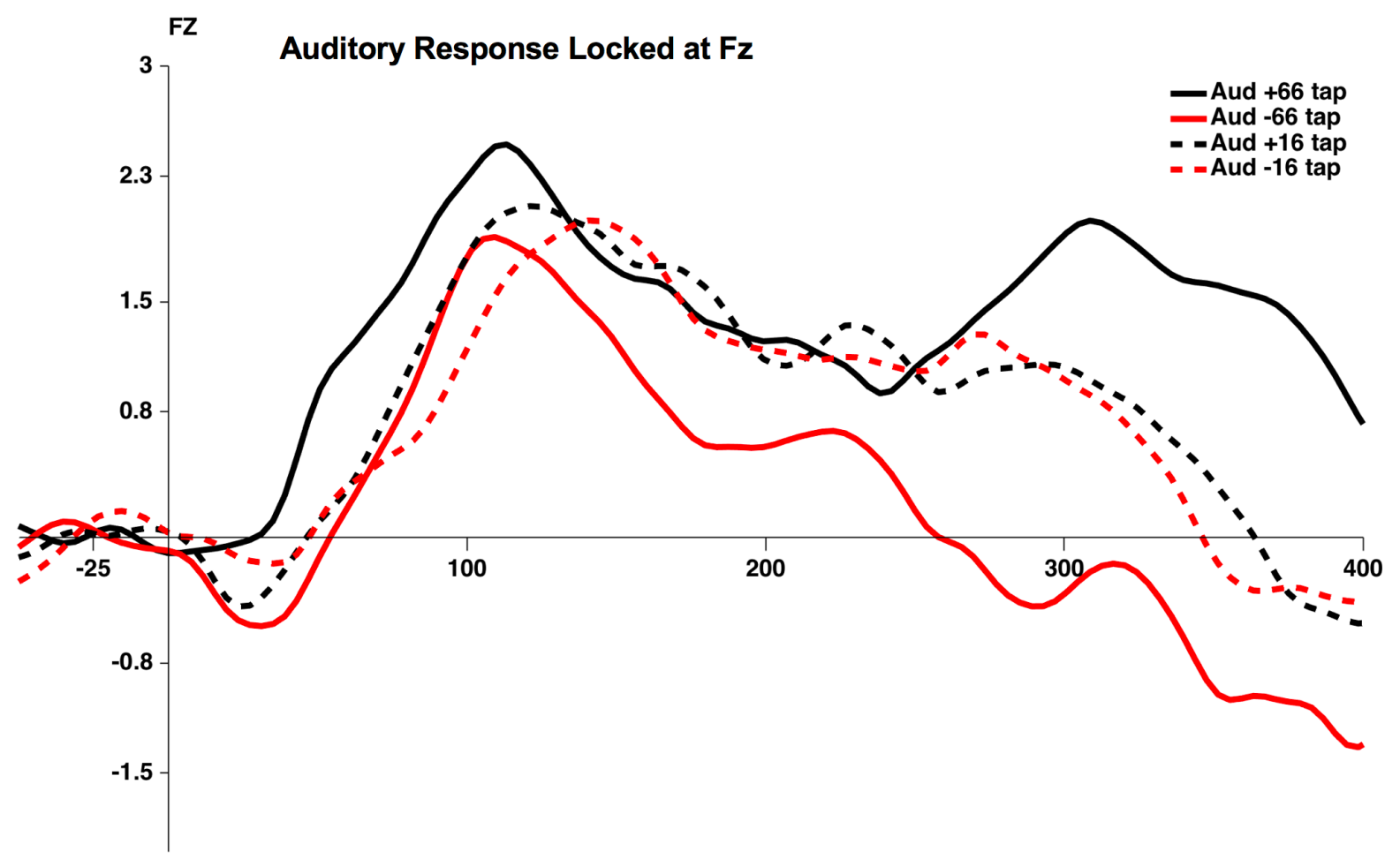

Figure 7. Response locked waveforms from time $\mathrm{T}$ for the 4 perturbation types from the Auditory condition.

The response-locked waveforms in the visual condition figure 8 (A) show a somewhat different looking waveform than compared to the auditory response-locked waveforms. This difference is due to the large frontal P3 component found in the visual stimulus locked potentials at Fz shown in figure $8 \mathrm{~B}$. The data were analyzed in the same fashion as the auditory response locked data. Once again in terms of the ERN, no significant effects were found, just as in the 
auditory data. In looking at the area of the Pe, a main effect was found of Direction $(1,9)=11.21$, $\mathrm{P}<.01$, but no significant effect of Magnitude was discovered. This finding corresponds to the fact that the large visual evoked frontal P3 component would show up in the response locked data shifted according to perturbation, and is not taken as an indication of any Pe or corresponding ERN.
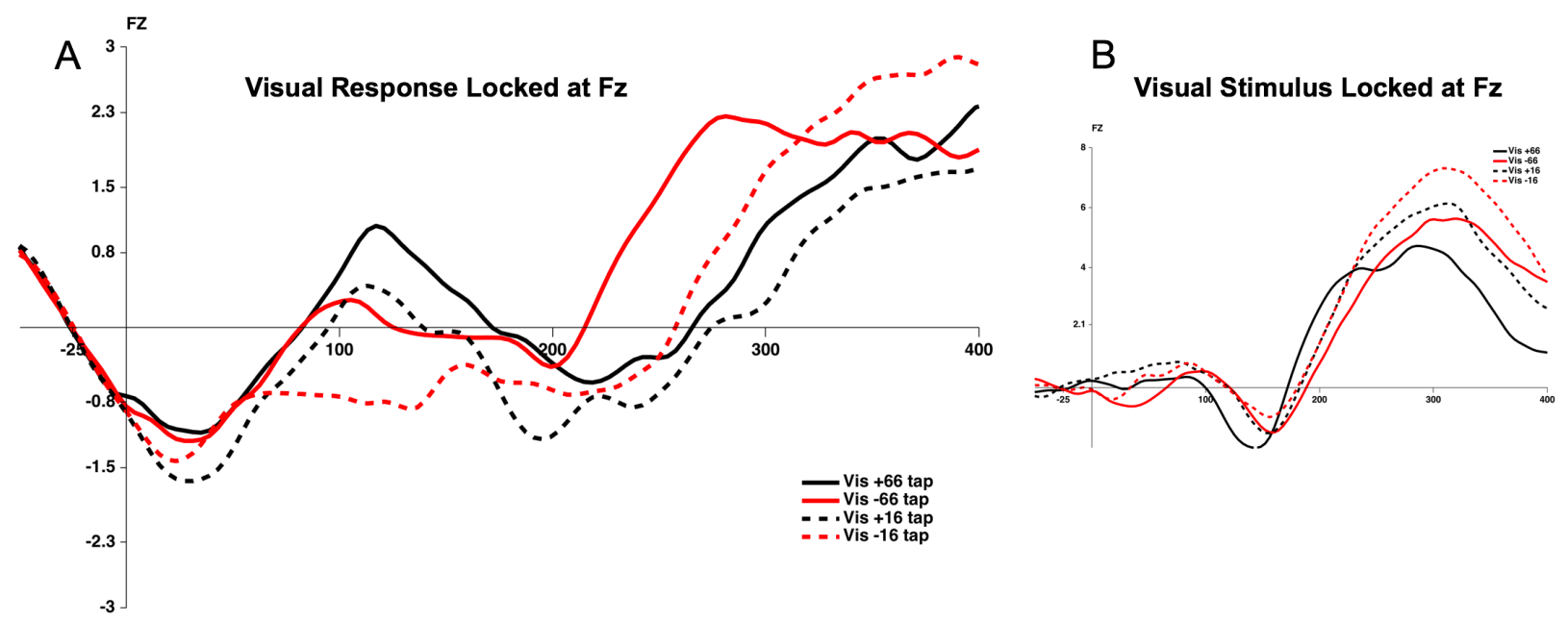

Figure 8. Visual condition waveforms at time $\mathrm{T}$ at Fz. (A) Visual response locked with the 4 perturbation types. Note the large late positive waveforms that differentiate in latency by perturbation condition. (B) Visual stimulus locked waveforms at Fz. Note the extremely large late waveforms. These waveforms explain the large late waveforms in (A)

\subsection{Source localization}

We investigated the underlying neural components of error detection and error correction using a clustering analysis of the dipoles localized from the individual independent components. The nature of dipole source localization on EEG data means that it does not have near the spatial precision as fMRI methods, however, when it is used in conjunction with evidence from other localization methods and neuroscience research it is a useful tool to determine the sources of the neural activity measured at the electrode level. The activity of the individual independent components with each cluster was averaged, resulting in an ERP for each cluster. This is to provide insight on the function of the components in relation to the ERPs generated at the scalp 
level. Our clustering analysis generated 12 clusters from 223 individual independent components left after artefactual components were rejected. Artefactual components consisting of blink and eye movement components were removed manually. Additionally, any component with a dipole residual variance greater than $15 \%$, and any component with a dipole outside of the brain were also removed from the analysis. We focus on 4 clusters here; 2 centered in the occipital lobe, one in the premotor cortex, and one focused at the anterior cingulate.

Looking at the clusters in the occipital lobe in figure 9, we see two similar components with one in the right occipital lobe and one in the left occipital lobe. The cluster in the right occipital lobe was consists of 19 independent components and the cluster in the left consists of 16 independent components. The ERPs in figure $9 \mathrm{C}$ and $\mathrm{D}$ are both time locked to the stimulus onset at time $\mathrm{T}$ for the $+/-66$ perturbations for both visual and auditory conditions. The ERPs generated are both consistent with the ERPs from the surface electrodes at $\mathrm{Oz}$, confirming that the visual evoked P1 originates in the visual cortex.
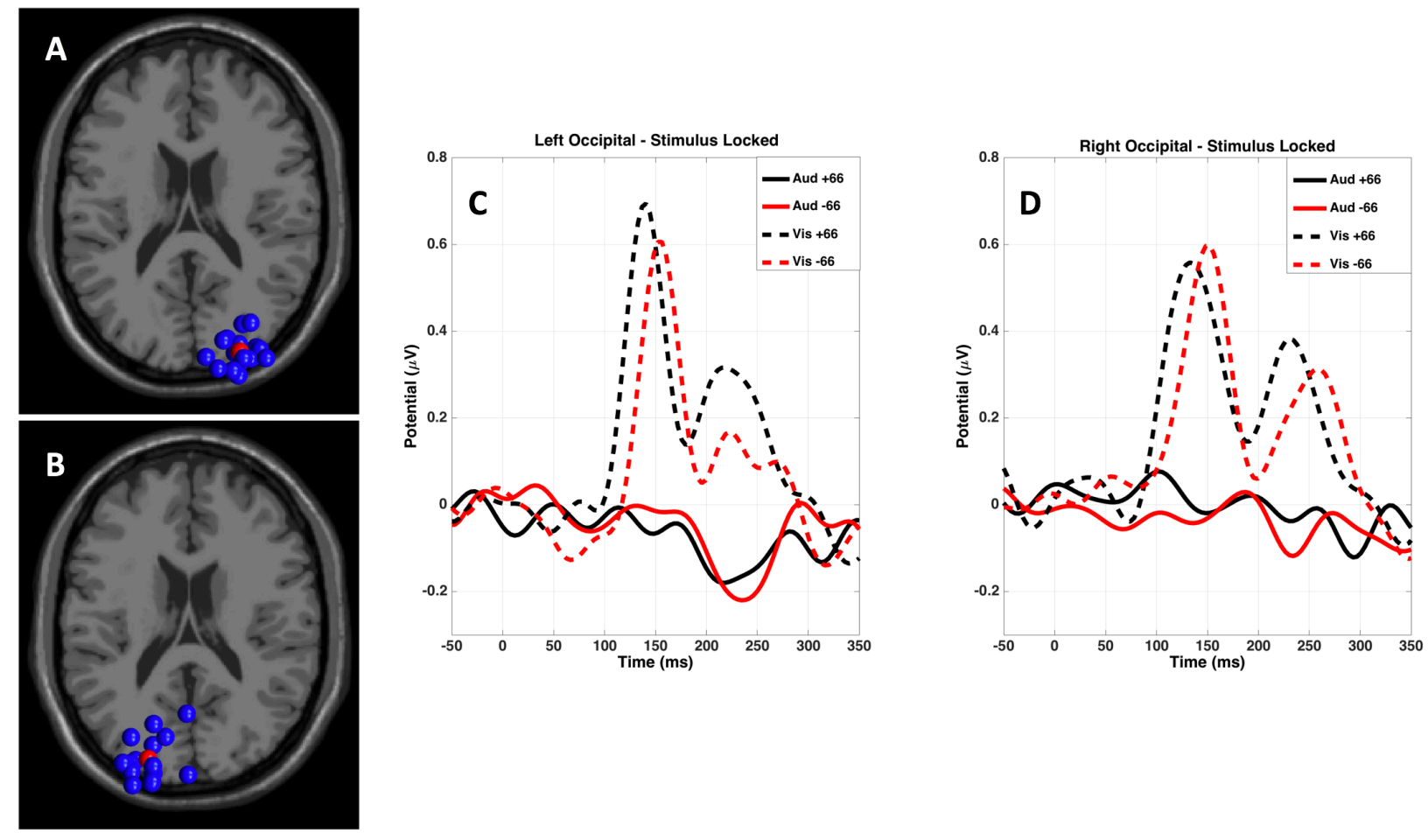
Figure 9. Clusters from the occipital lobe and their associated stimulus locked ERPs. (A) Cluster in the right Occipital Lobe. (B) Cluster in the left Occipital Lobe. (C) Stimulus locked ERP from the left Occipital cluster at time $\mathrm{T}$ for the $+/-66 \mathrm{~ms}$ perturbations for both Auditory and Visual conditions. (D) Stimulus locked ERP from the right Occipital cluster at time $\mathrm{T}$ for the $+/-66 \mathrm{~ms}$ perturbations for both Auditory and Visual conditions.

The cluster centered on the premotor cortex in figure 10 consists of 19 independent components. We generated 2 ERPs from components in this cluster; one time locked to the stimulus at time $\mathrm{T}$ (figure $10 \mathrm{~B}$ ), and one time locked to the tap onset at time $\mathrm{T}$ (figure $10 \mathrm{C}$ ). Both ERPs contain the +/- 66 perturbations for both auditory and visual conditions. For the Auditory stimulus locked ERP, we find negative going waveforms peaking at approximately 130 ms post stimulus onset and a following positive waveform peaking around $190 \mathrm{~ms}$ in the +66 condition and near $240 \mathrm{~ms}$ in the -66 condition. These peaks correspond roughly with Auditory $\mathrm{N} 1$ and P2 waves found in the channel electrodes at Fz. No such waveforms are found in the visual stimulus locked ERPs, suggesting that the premotor cortex may be playing a role in auditory error detection but not in visual error detection. The response locked ERPs show a premovement negativity for both Auditory and Visual conditions, and large post-movement positivity around $100 \mathrm{~ms}$ in both Auditory conditions, with a smaller post-movement positivity in the visual conditions. 

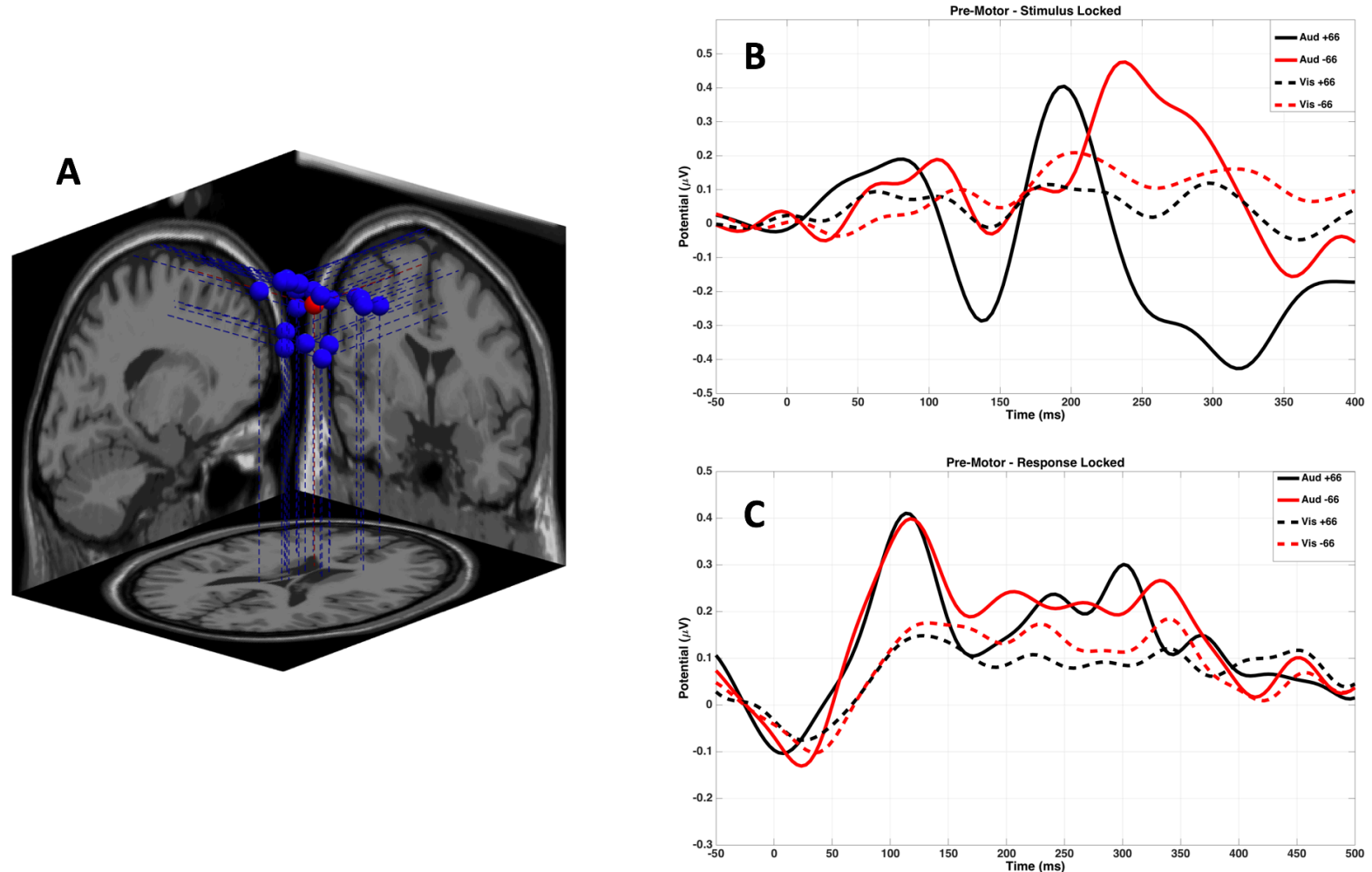

Figure 10. Cluster centered around the pre-motor cortex and associated ERPs. (A) Clustered component dipoles centered approximately at 90the pre-motor cortex (B) Stimulus locked ERP from pre-motor cluster at time $\mathrm{T}$ for the $+/-66 \mathrm{~ms}$ perturbations for both Auditory and Visual conditions. (C) Response locked ERP from pre-motor cluster at time $\mathrm{T}$ for the $+/-66 \mathrm{~ms}$ perturbations for both Auditory and Visual conditions.

The cluster centered approximately on the anterior cingulate shown in figure 11 consists of 19 independent components. We generated 2 ERPs from this cluster; one time locked to the stimulus at time $\mathrm{T}$ (figure $11 \mathrm{~B}$ ), and one time locked to the tap onset at time $\mathrm{T}$ (figure $11 \mathrm{C}$ ). Both ERPs contain the +/- 66 perturbations for both auditory and visual conditions. In the stimulus locked ERP, we see a waveform strongly resembling those detected at Fz in the auditory conditions (shown in figure 5) with similar auditory N1 and P2 peak latencies, showing a role for the anterior cingulate in auditory error detection. In the visual stimulus locked waveforms a negative wave with a peak near 150 ms post matches the anterior N1 measured at Fz for the visual stimulus shown in figure $8 \mathrm{~B}$, suggesting that there is some role for the anterior 
cingulate in error detection for visual stimuli. Looking at the response locked waveforms there is a pre-movement negativity followed by a post-movement positivity peaking near $100 \mathrm{~ms}$ post tap for both Auditory and Visual conditions. In the Auditory +66 there is an additional peak near 330 ms, but none for the Auditory -66 condition or for either visual condition. This peak corresponds with the Pe found in the Auditory response locked waveforms at Fz, suggesting a further role for anterior cingulate in error detection and correction.
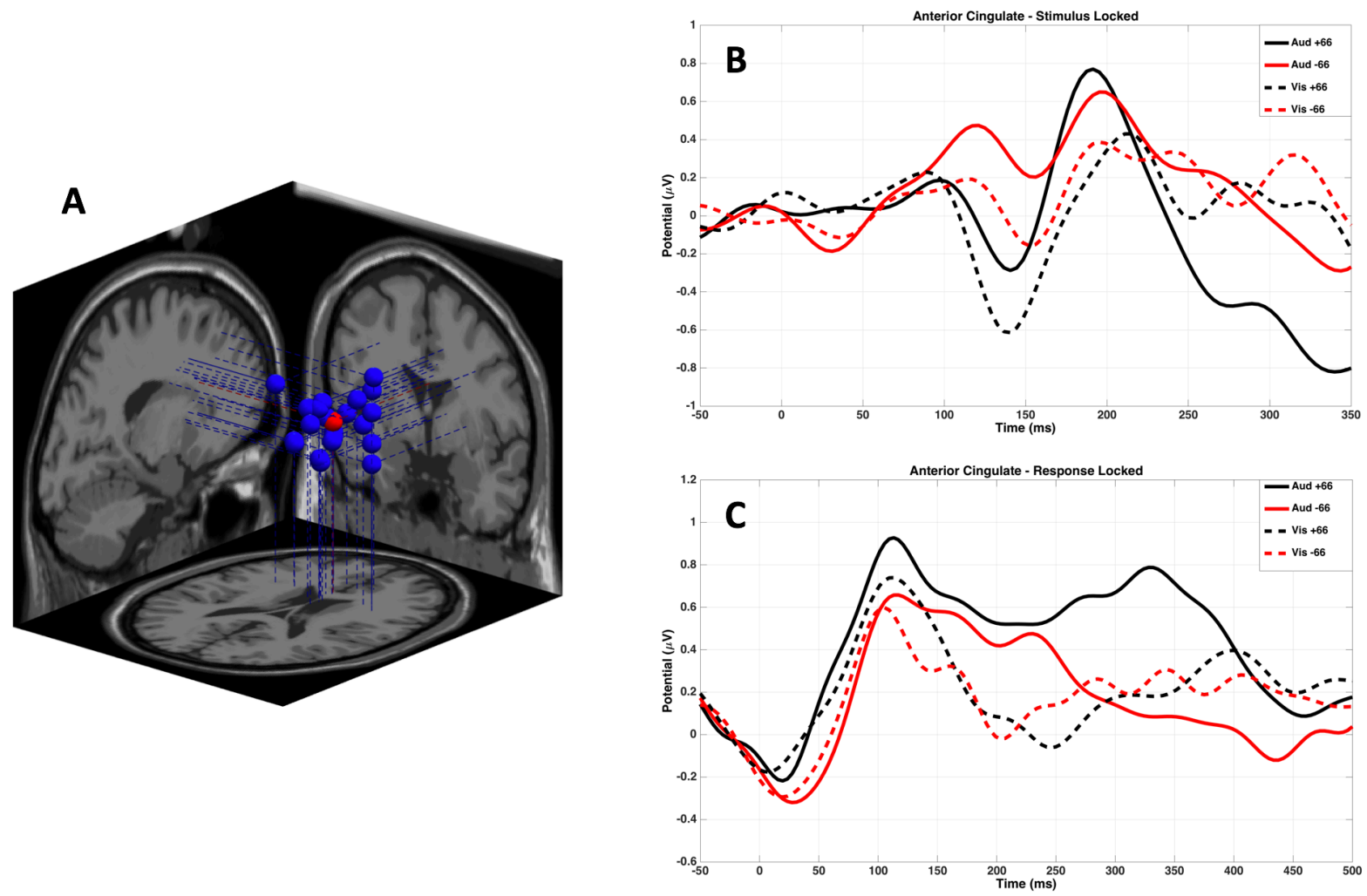

Figure 11. Cluster centered around the anterior cingulate and associated ERPs. (A) Clustered component dipoles centered approximately at the anterior cingulate. (B) Stimulus locked ERP from anterior cingulate cluster at time $\mathrm{T}$ for the $+/-66 \mathrm{~ms}$ perturbations for both Auditory and Visual conditions. (C) Response locked ERP from anterior cingulate cluster at time $\mathrm{T}$ for the $+/-66 \mathrm{~ms}$ perturbations for both Auditory and Visual conditions. 


\section{Discussion}

\subsection{Summary of results}

Our data show clear differences in error correction between auditory and visual sensorimotor synchronization. Within the tapping data, we show that changes in perturbation direction or magnitude do not affect the rate of correction in the visual condition. In the auditory condition, we find clear differences in rate of correction with the direction of the perturbations, which is largely attributed to the pronounced under- and overcorrection. Within both the AEPs and VEPs we find that the +66 perturbations produce the largest effect. In the auditory condition we see pronounced N1 component for the +66 condition at Fz, and in the visual condition we find a reduced latency for the visual P1 component at Oz. The -66 condition produced what may be a MMN in the Auditory -66 condition, while the visual -66 condition resulted in a larger N1/attenuated P2 component. Looking at the response locked waveforms we only find evidence suggesting an ERN in the Auditory +66 with the pronounced error positivity $(\mathrm{Pe})$. The localization results suggest that the visual system is performing at least some of the timing activity of rhythm perception within the visual cortex, while the auditory timing of rhythm perception appears to be performed within the motor cortex. Additionally, the anterior cingulate cortex is implicated in error monitoring for both the incoming stimuli and for errors made in response to perturbed stimuli.

\subsection{Differences between auditory and visual behavioral data}

The normalized asynchrony data show the visual correction is happening at the same rate regardless of the size or direction of the error. The gradual undifferentiated correction in the visual tapping data suggests that the visual system is only using a phase correction in response to 
the perturbations. The auditory data, on the other hand, show an effect of the direction in the normalized asynchrony of the data, which we suggest is due to the amount of undercorrection for the late coming perturbations and overcorrection for the early coming perturbations. While our data does not show a clear effect of the size of the perturbation on correction for auditory stimuli, several previous studies have shown this effect, suggesting a difference in phase correction and period correction (Repp, 2000 \& 2001; Praamstra et al, 2003). While our data do not clearly show the classical pattern of period correction, we suggest that the evidence from previous research shows the perturbations of $+/-66 \mathrm{~ms}$ do induce period corrections.

\subsection{Stimulus locked ERPs}

The auditory evoked potential (AEP) of focus of this study is the N1, which is believed to signal detection of changes in the auditory environment (Hyde, 1997), and is linked to the modulation of attention such that an increase in N1 amplitude links with increased attention (Lange 2013). The AEPs show a consistent pattern where the $+/-16 \mathrm{~ms}$ perturbations produce no significant differences in wave forms, which suggests that the mechanisms connected to the auditory N1 are not sensitive to small phase correction inducing shifts, and therefore do not help to modulate attention in order to correct errors. Consistent with the findings in Praamstra et al., (2003), the larger magnitude errors of $+/-66 \mathrm{~ms}$ provoke effects on the N1 by increasing the N1 amplitude and thus suggest period correction is tied to the mechanisms responsible for the N1. In addition to the effects on the N1, in the -66 perturbation we see a wave form that may be attributed to a Mismatch Negativity (MMN), which is thought to be a pre-attentional response to an auditory change great enough to affect a pertinent behavioral activity (Näätänen, \& Winkler, 1999). Previous research has shown that the MMN can be activated in response to an 
unexpectedly early tone in otherwise isochronous rhythms ( Ford \& Hillyard, 1981; Rüsseler et al, 2001). These effects suggest there may be different mechanisms of error direction depending on the direction of error.

The visual evoked potential (VEP) of focus for this study is the visual P1, which originates in the occipital lobe and is believed to be due to inhibitory processes resulting from thalamic input to the visual cortex (Kraut et al, 1985). The P1 is effected by changes in luminance, such as flashes. Additionally, the latency of the P1 peak has been shown to decrease in response to large changes in amount of luminance (Luck \& Kappenman, 2011). Our data show the P1 was not responsive to any of the perturbations except for the +66 condition, and only by way of a reduction in peak latency. The fact that the latency was reduced in the large, late coming perturbations suggests that the P1 mechanism was primed or expecting the onset of the stimulus. This indicates that a measure rhythmic timing may be occurring within the visual cortex.

\subsection{Subcortical and cortical processes}

In both the auditory and visual conditions, the evoked potentials only show responses to the large perturbations: both + and -66 perturbations induced larger N1 amplitudes in the auditory condition, and the +66 condition reduced the latency of $\mathrm{P} 1$ in the visual condition. These evoked potentials originate in the cortex since the subcortical regions of the brain do not have the geometric neural alignment necessary to evoke event related potentials. Indeed, our source localization data suggest that the anterior cingulate is directly involved in the periodcorrection process, as would be expected from the known error monitoring role of the anterior cingulate (Botvinick et al, 2001 \& 2004; Ridderinkhof et al, 2004). So, while our data show that 
at least some of the processing of period-correction errors is happening at the cortical level, any phase correction processing is likely happening at the subcortical level, with previous research perhaps suggesting that the basal ganglia might play a large role in this process (Cameron et al, 2016; Grahn \& Brett, 2009).

\subsection{The role of the $E R N$}

The ERN is known to arise in the result of making an error, even when that error is not reported. (Falkenstein et al, 2000; Olvet \& Hajcak, 2008) The error positivity (Pe) often follows the ERN, but only when that error is consciously detected (Overbeek et al, 2005; Endrass et al, 2007). Although our data do not show a statistically significant ERN, they are suggestive of its existence as backed up by the large Pe found in the auditory +66 condition. This is similar to what Praamstra et al., (2003) found where only the auditory +50 condition elicited an ERN in their study. The fact that this error monitoring activity only arose from the large, late-arriving perturbation is likely due to the fact that synchronized tapping to auditory tones usually involves tapping roughly $50 \mathrm{~ms}$ before the actual tone onset, or what is known as the negative mean asynchrony (Repp, 2005). Since the subjects are tapping slightly ahead of the expected onset, a late tone onset will seem slightly more deviant than it actually is, while an early tone onset will seem less deviant. In the case of the visual condition, there is a lack of evidence for the ERN which suggests there was simply not enough temporal acuity provided by the visual system to engage this error mechanism.

\subsection{Source analysis}

The clustering analysis done on the component dipoles suggest the visual system is processing the timing of the visual rhythmic flashes at some level in the visual cortex, as well as 
within the anterior cingulate. When looking at the timing of the evoked potentials between the visual cortex and the anterior cingulate for the large perturbations, we can see that both show large waveforms peaking at approximately the same point in time, suggesting that the two areas are tied together in the rhythmic timing and error monitoring of the visual flashes. We also find evidence that the anterior cingulate is involved in the error monitoring of the auditory stimuli as well as for monitoring response errors in tapping timing. This role of the anterior cingulate in error monitoring for both stimulus and response errors fits with the perceived role of the anterior cingulate as implicated in cognitive control functions that allow the brain to adapt behavior to changing task demands and circumstances (Botvinick et al., 2001 \& 2004; Ridderinkhof et al., 2004)

\subsection{Visual error correction mechanism}

Many previous studies have shown that the auditory system is tightly coupled with the motor system for beat perception (Chen et al., 2006 \& 2008; Grahn \& Brett, 2007; Grahn \& Rowe, 2009). This coupling with the motor system results in strong synchronization abilities to auditory rhythms. The visual system, however, is not able to provide the necessary information to the motor system to allow for motor synchronization to flashing visual rhythms with the same level of accuracy seen with auditory rhythms. It is known that synchronization ability to a moving visual rhythm, e.g. a bouncing ball, is much closer to the ability to synchronize to an auditory rhythm (Hove et al., 2010 \& 2013), although it is not clear that synchronizing movements to a moving stimulus is invoking a sense of rhythm in the same way as an auditory rhythm. Likewise, synchronizing to an auditory rhythm that consists of a frequency modulated siren also results in reduced synchronization ability compared to a normal rhythm (Hove et al., 
2013). Neither of these kinds of stimuli have been used in an error correction task, so the capabilities of error correction for these stimuli remain unknown. We do see evidence that the visual system can detect at least some errors with flashing stimuli based on the latency reduction of the visual P1 to the +66 perturbations. Curiously, even though the visual system is detecting this error, it does not lead to any improvement in synchronization error correction. This suggests the visual system is simply not coupled to the motor system for rhythm entrainment in the same way that the auditory system is, and therefore explains why synchronization to flashing visual rhythms is much more difficult than synchronization to a similar auditory rhythm.

\subsection{Conclusion}

There are several key findings in this study. The suggestion that there is a MMN response for the -66 auditory condition implies there may be separate mechanisms in play for detecting errors, depending on the direction of the errors. We also find that the anterior cingulate is implicated in error monitoring processes for the rhythmic timing of incoming stimuli as well as for responses in synchronization induced by perturbations in the otherwise isochronous rhythm. The lack of evidence for neural correlates of phase correction suggests that the processes involved in phase correction are subtle and likely subcortical. Finally, the fact that it is difficult to entrain to a visual flashing rhythm is likely due to the visual system not being coupled to the motor system as strongly as the auditory system. It is also possible that there are separate mechanisms of visual synchronization, but this subject matter needs more study before those mechanisms may be determined. 


\section{References}

Balasubramaniam, R., Wing, A. M., \& Daffertshofer, A. (2004). Keeping with the beat: movement trajectories contribute to movement timing. Experimental Brain Research, 159(1), 129-134.

Botvinick, M. M., Braver, T. S., Barch, D. M., Carter, C. S., \& Cohen, J. D. (2001). Conflict monitoring and cognitive control. Psychological review, 108(3), 624.

Botvinick, M. M., Cohen, J. D., \& Carter, C. S. (2004). Conflict monitoring and anterior cingulate cortex: an update. Trends in cognitive sciences, 8(12), 539-546.

Cameron, D. J., Pickett, K. A., Earhart, G. M., \& Grahn, J. A. (2016). The effect of dopaminergic medication on beat-based auditory timing in Parkinson's disease. Frontiers in neurology, 7.

Chen, R., Yaseen, Z., Cohen, L. G., \& Hallett, M. (1998). Time course of corticospinal excitability in reaction time and self-paced movements. Annals of neurology, 44(3), 317-325.

Chen, J. L., Zatorre, R. J., \& Penhune, V. B. (2006). Interactions between auditory and dorsal premotor cortex during synchronization to musical rhythms. Neuroimage, 32(4), 1771-1781.

Chen, J. L., Penhune, V. B., \& Zatorre, R. J. (2008). Listening to musical rhythms recruits motor regions of the brain. Cerebral cortex, 18(12), 2844-2854.

Collective, B. S. M., \& Shaw, D. (2012, February). Makey Makey: improvising tangible and nature-based user interfaces. In Proceedings of the sixth international conference on tangible, embedded and embodied interaction (pp. 367-370). ACM.

Delorme, A., \& Makeig, S. (2004). EEGLAB: an open source toolbox for analysis of single-trial EEG dynamics including independent component analysis. Journal of neuroscience methods, 134(1), 9-21.

Endrass, T., Reuter, B., \& Kathmann, N. (2007). ERP correlates of conscious error recognition: aware and unaware errors in an antisaccade task. European Journal of Neuroscience, 26(6), 1714-1720.

Falkenstein, M., Hoormann, J., Christ, S., \& Hohnsbein, J. (2000). ERP components on reaction errors and their functional significance: a tutorial. Biological psychology, 51(2), 87-107.

Ford, J. M., \& Hillyard, S. A. (1981). Event-Related Potentials (ERPs) to Interruptions of a Steady Rhythm. Psychophysiology, 18(3), 322-330.

Grahn, J. A., \& Brett, M. (2007). Rhythm and beat perception in motor areas of the brain. Journal of cognitive neuroscience, 19(5), 893-906.

Grahn, J. A., \& Brett, M. (2009). Impairment of beat-based rhythm discrimination in Parkinson's disease. Cortex, 45(1), 54-61.

Grahn, J. A., \& Rowe, J. B. (2009). Feeling the beat: premotor and striatal interactions in musicians and nonmusicians during beat perception. Journal of Neuroscience, 29(23), 7540-7548.

Hove, M. J., Fairhurst, M. T., Kotz, S. A., \& Keller, P. E. (2013). Synchronizing with auditory and visual rhythms: an fMRI assessment of modality differences and modality appropriateness. Neuroimage, 67, 313-321.

Hove, M. J., Iversen, J. R., Zhang, A., \& Repp, B. H. (2013). Synchronization with competing visual and auditory rhythms: bouncing ball meets metronome. Psychological Research, 77(4), 388-398.

Hove, M. J., Marie, C., Bruce, I. C., \& Trainor, L. J. (2014). Superior time perception for lower musical pitch explains why bass-ranged instruments lay down musical rhythms. Proceedings of the National Academy of Sciences, 111(28), 10383-10388. 
Hove, M. J., Spivey, M. J., \& Krumhansl, C. L. (2010). Compatibility of motion facilitates visuomotor synchronization. Journal of Experimental Psychology: Human Perception and Performance, 36(6), 1525.

Jang, J., Jones, M., Milne, E., Wilson, D., \& Lee, K. H. (2016). Contingent negative variation (CNV) associated with sensorimotor timing error correction. NeuroImage, 127, 58-66.

Kavanagk, R. N., Darcey, T. M., Lehmann, D., \& Fender, D. H. (1978). Evaluation of methods for threedimensional localization of electrical sources in the human brain. IEEE Transactions on Biomedical Engineering, (5), 421-429.

Kraut, M. A., Arezzo, J. C., \& Vaughan, H. G. (1985). Intracortical generators of the flash VEP in monkeys. Electroencephalography and Clinical Neurophysiology/Evoked Potentials Section, 62(4), 300-312.

Lange, K. (2013). The ups and downs of temporal orienting: a review of auditory temporal orienting studies and a model associating the heterogeneous findings on the auditory N1 with opposite effects of attention and prediction. Frontiers in human neuroscience, 7.

Lopez-Calderon, J., \& Luck, S. J. (2014). ERPLAB: an open-source toolbox for the analysis of eventrelated potentials. Frontiers in human neuroscience, 8.

Luck, S. J., \& Kappenman, E. S. (Eds.). (2011). The Oxford handbook of event-related potential components. Oxford university press.

Mullen, T. R., Kothe, C. A., Chi, Y. M., Ojeda, A., Kerth, T., Makeig, S., ... \& Cauwenberghs, G. (2015). Real-time neuroimaging and cognitive monitoring using wearable dry EEG. IEEE Transactions on Biomedical Engineering, 62(11), 2553-2567.

Näätänen, R., \& Winkler, I. (1999). The concept of auditory stimulus representation in cognitive neuroscience. Psychological bulletin, 125(6), 826.

Olvet, D. M., \& Hajcak, G. (2008). The error-related negativity (ERN) and psychopathology: toward an endophenotype. Clinical psychology review, 28(8), 1343-1354.

Overbeek, T. J., Nieuwenhuis, S., \& Ridderinkhof, K. R. (2005). Dissociable components of error processing: on the functional significance of the Pe vis-à-vis the ERN/Ne. Journal of Psychophysiology, 19(4), 319-329.

Praamstra, P., Turgeon, M., Hesse, C. W., Wing, A. M., \& Perryer, L. (2003). Neurophysiological correlates of error correction in sensorimotor-synchronization. Neuroimage, 20(2), 1283-1297.

Scherg, M. (1990). Fundamentals of dipole source potential analysis. Auditory evoked magnetic fields and electric potentials. Advances in audiology, 6, 40-69.

Repp, B. H. (2000). Compensation for subliminal timing perturbations in perceptual-motor synchronization. Psychological research, 63(2), 106-128.

Repp, B. H. (2001). Processes underlying adaptation to tempo changes in sensorimotor synchronization. Human movement science, 20(3), 277-312.

Repp, B. H. (2005). Sensorimotor synchronization: a review of the tapping literature. Psychonomic bulletin \& review, 12(6), 969-992.

Repp, B. H., \& Keller, P. E. (2004). Adaptation to tempo changes in sensorimotor synchronization: Effects of intention, attention, and awareness. Quarterly Journal of Experimental Psychology Section A, 57(3), 499-521.

Repp, B. H., \& Su, Y. H. (2013). Sensorimotor synchronization: a review of recent research (20062012). Psychonomic bulletin \& review, 20(3), 403-452. 
Ridderinkhof, K. R., Ullsperger, M., Crone, E. A., \& Nieuwenhuis, S. (2004). The role of the medial frontal cortex in cognitive control. Science, 306(5695), 443-447.

Rüsseler, J., Altenmüller, E., Nager, W., Kohlmetz, C., \& Münte, T. F. (2001). Event-related brain potentials to sound omissions differ in musicians and non-musicians. Neuroscience letters, 308(1), 33-36.

Thaut, M. H., Miller, R. A., \& Schauer, L. M. (1998). Multiple synchronization strategies in rhythmic sensorimotor tasks: phase vs period correction. Biological cybernetics, 79(3), 241-250.

Vorberg, D., \& Wing, A. (1996). Modeling variability and dependence in timing. Handbook of perception and action, 2, 181-262.

Yeung, N., Botvinick, M. M., \& Cohen, J. D. (2004). The neural basis of error detection: conflict monitoring and the error-related negativity. Psychological review, 111(4), 931. 


\section{Acknowledgments}

This work was supported by an NSF grant BCS 1460633 awarded to the corresponding author (RB). DCC and RB were also supported by an award from the Office of the President, University of California for the UC-MERCI initiative awarded to Scott Makeig (PI). We thank Scott Makeig, John Iversen, and members of the Sensorimotor Neuroscience Laboratory at UC Merced and the Swartz Center for Computational Neuroscience at UC San Diego for their thoughtful advice and guidance. 